\title{
A Calculation Theory of Earth Pressure Considering Microstructure of Porous Materials
}

\author{
Jun-hu $\mathrm{Li}^{1 *}$, Chao Zhang ${ }^{2}$, Wei Xue ${ }^{3,4}$, Wen-chao Zhang ${ }^{3,4}$, and Ri-qing Xu ${ }^{1}$ \\ ${ }^{1}$ Zhejiang University, Research Center of Coastal and Urban Geotechnical Engineering, 310058 \\ Hangzhou, China \\ ${ }^{2}$ China Road and Bridge Corporation, 100011 Beijing, China \\ ${ }^{3}$ Guangzhou Chemical Grouting Engineering Co., Ltd., CAS, 510650 Guangzhou, China \\ ${ }^{4}$ Guangdong Province Chemical Grouting Engineering and Materials Academician Workstation, CAS, \\ 510650 Guangzhou, China
}

\begin{abstract}
To explore a more reasonable earth pressure calculation theory, microstructure characteristics of soft clay in Hangzhou, Ningbo and Wenzhou had been observed using scanning electron microscope (SEM) and were quantitatively analysed using Image-Pro Plus (IPP) software. The relationship between porosity and contact area of soft clay was got by considering the microstructure. Combining soil lateral stress transfer principle, a calculation theory of earth pressure considering soil contact area was got. The possible reason of the differences between earth pressure and the actual monitoring earth pressure was analysis by the case.
\end{abstract}

\section{Introduction}

The calculation of earth pressure is one of the primary research topics of soil mechanics. The method of estimating water and earth pressures together or separately can't simply meet the needs of engineering, while these two methods are still under discussion. With the development of urban construction and the exploitation of the underground space, especially the construction of large underground emporium and subway tunnels, there is growing concern about the working condition of different kinds of supporting structure during the design and construction. Anecdotal evidence suggests that the interaction between the soil and the supporting structures is much more difficult than what we have learnt, and the method to calculate the earth pressure on the back the soil retaining structures is still under discussion.

As for these questions, the scholars domestic and abroad have carried out plenty of qualitative and quantitative research work on microstructure of fine particles based on microscope images in the past few decades [1-5]. Chen Yu-jiong [6] believed that, as far as the method of estimating water and earth pressures together manages to avoid the puzzle of the pore water pressure by seeing the water and soil as an integral using total stress strength parameters, there is no need to figure out the mechanical mechanism of the pore water pressure.

* Corresponding author: 285014418@.qq.com 
But Li Guang-xin [7] pointed out the insufficiency of this method and believed that this method makes sense from microcosmic point of view. Besides, he proposed to incorporate the view of microstructure into the study of stress transmission mechanism. On the basis of previous studies, the paper analyses soft clay microstructure and modifies the commonly used formula of earth pressure, followed by a calculation theory considering soil contact area.

\section{The relationship between soil contact area and porosity}

\subsection{The micro porosity of the clay}

Fig.1. is the microscopic pore figure of some kind of soft clay observed using scanning electron microscope (SEM). We can see from this figure that the gray level of the pore of soft clay decreases from deep to shallow. In the bottom of the pore, the gray level is highest and the color is darkest, which is opposite on the opening of the pore. The sample used in experiments shows the section area is getting bigger from the bottom to opening.

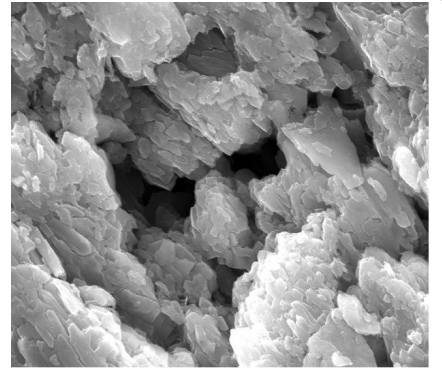

Fig.1. Microscopic pore figure of soft clay

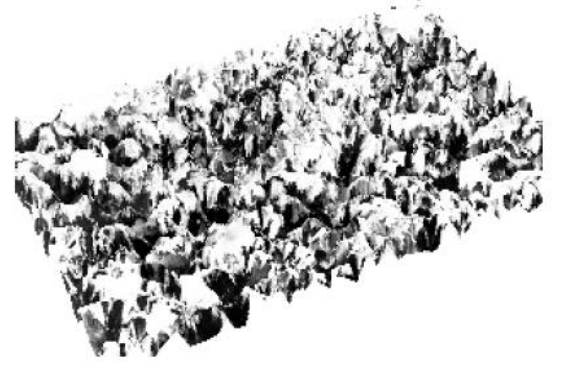

Fig.2. Three-dimensional display of particle surface

In the SEM figure, the pixel represents the area of the figure and the gray level stands for the colour depth. Different gray levels can be expressed by different thresholds, of which the values range from 0 to 255 . We set the brightness of the deepest place as the minimum value, and when the threshold goes larger, the brightness varies from deep to shallow.

Wang Bao-jun [8], basing on the gray level, with the help of ArcGIS software, turns the SEM figure into the three-dimensional display image of particle surface which shows the 3D distribution of the pore in the soil. In the three-dimensional space model based on this idea, which is shown in fig.3., the area of plan in the horizontal direction can be expressed by the pixels, while the vertical height can be characterized by the threshold.

In order to calculate the volume of the irregular shape, we apply the means of integration. The volume can be got by multiplying the area of each section by the corresponding height. The different thresholds in the SEM figure correspond to the area of pores on different section. If we make the threshold as small as possible, the volume of the irregular shape can be obtained. Symbol Yi stands for different thresholds. Suppose the area of the smaller loop at the gray level of threshold Yi is $A_{i}$, the volume of pore between these two thresholds is equal to:

$$
\begin{gathered}
\Delta V_{i}=\left(\frac{A_{i+1}+A_{i}}{2}\right) \times\left(\mathrm{Y}_{i+1}-\mathrm{Y}_{i}\right) \\
d V=\Delta V_{i}
\end{gathered}
$$


According to the calculus theory, when the value of $\Delta Y_{i}$ (i.e. $Y_{i+1}-Y_{i}$ ) is small enough, the values of $A_{i+1}$ and $A_{i}$ will be extremely close. This time, the pore volume calculated will extremely approximates the real volume. This method will be expected to have similar satisfactory result, if it is generalized into all the pores (as a whole). The total volume of the pores can be expressed by:

$$
V_{3 D}=\sum_{i=1}^{255}\left(\frac{A_{i+1}+A_{i}}{2}\right) \times\left(\mathrm{Y}_{i+1}-\mathrm{Y}_{i}\right)
$$

Also, we can get the 3D porosity at arbitrary thresholds of gray level:

$$
n_{3 D}=\frac{\sum_{i=1}^{m}\left(\frac{A_{i}+A_{i+1}}{2}\right) \times\left(\mathrm{Y}_{i+1}-\mathrm{Y}_{\mathrm{i}}\right)}{\left(Y_{m}-Y_{0}\right) \times S_{A}}
$$

In this formula, $m$ is the value of thresholds and $S_{A}$ is the area of the pixels of selected regions, which is analyzed by Image-Pro Plus (IPP) software. The initial threshold $\mathrm{Y}_{0}$ is equal to 0 .

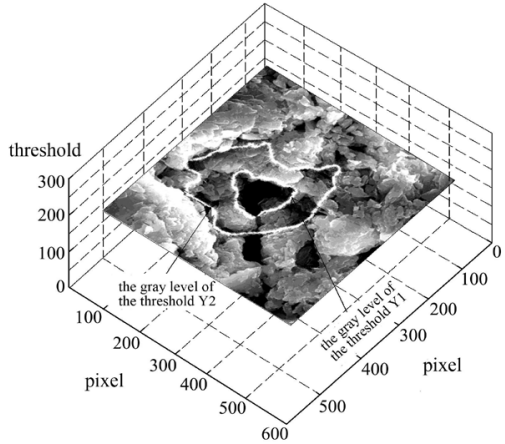

Fig.3. Calculate model of 3D porosity

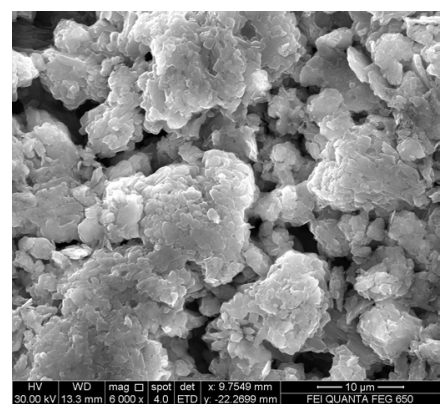

Fig.4. Soil particles contact case

\subsection{The rate of the contact area of clay particles}

In principle of effective stress based on porous medium, Xu Xian-zhi [9] pointed out that the contact area of the soil particles is far less than that of the soil mass, which may apply to the soil with macropores or point-contacting particles. As for the soil with low porosity which has different kinds of contacting patterns of the soil particles, the contact area should not be ignored. Obviously, whether this conjecture is reasonable depends on the microstructure of the soil. In the microstructure figure of the soft clay shown as fig.4., contacting patterns of the soil particles is not merely point-contacting and not all porosities of the soil are large. So it's necessary to make further research to find out what the real contact area is, with deep insights into facts of contacting patterns of the soil particles.

Utilizing the statistical method presented in the paper, the 3D porosity of the micro figure can be obtained, comparing with the macro porosity which is got from the soil laboratory tests. When the values of these two porosities are the same, we define the corresponding pixel of the gray level analysis in the IPP figure as the contact area. And the ratio of the area of pixel to the total area of the pixel is defined as the rate of the average contact area $\left(R_{C A}\right)$. 


\subsection{The relationship between the contact area and porosity}

According to the method mentioned above, we carried out an experiment by scanning the soft clay from three cities (Hangzhou, Ningbo and Wenzhou) and got 225 SEM images analyzed, accomplished by configuring the moisture content of the soil to control the size of the porosity. The rate of the average contact area can be expressed by the statistical mean of 5 SEM images value from each test specimen with different porosity. We can get the relationship of the contact area $\left(A_{C}\right)$, pore area $\left(A_{P}\right)$ and total area $(A)$ as following:

$$
\frac{A_{C}}{A}+\frac{A_{P}}{A}=1
$$

Assuming $\frac{A_{C}}{A}=R_{C A}, \quad R_{C A}$ is the rate of the average contact area, based on average conception. The contact area includes the point contact, line contact and surface contact. Besides we define $\frac{A_{P}}{A}=R_{P A}$ as the rate of the average pore area.

Since the porosity and the rate of the average pore area are both representations of the soil pores, we can establish their relation as $R_{P A}=f(n)$. Then we can get the rate of the average contact area as following:

$$
R_{C A}=1-f(n)
$$

In practical situation, the soil is in a humid environment and the double electric layer on the surface of the clay particle includes the strong and weak bound water. The water film thickness of montmorillonite is $210 \AA$ (or Angstrom), and that of kaolin is $410 \AA$. The hydrophilic property of illitic soil is somewhere in between both of those, so its water film thickness ranges between $210 \AA$ and $410 \AA$.

According to the definition of the length, we get $1 \AA=10^{-10} \mathrm{~m}=10^{-4} \mu \mathrm{m}$. On the principle of the microcosmic measurement, the length of 1 pixel is equal to $3.448 \mu \mathrm{m}$ when the amplification factor is 100 (Chen Cui-cui [10]). In the study, the amplification factor is set as 1000 , so the length of 1 pixel is equal to $0.3448 \mu \mathrm{m}$. In fig. 5 ., we can get the contact area of the clay considering the water film thickness:

$$
A_{C}=A+S \times \frac{H \times 10^{-4}}{0.3448}
$$

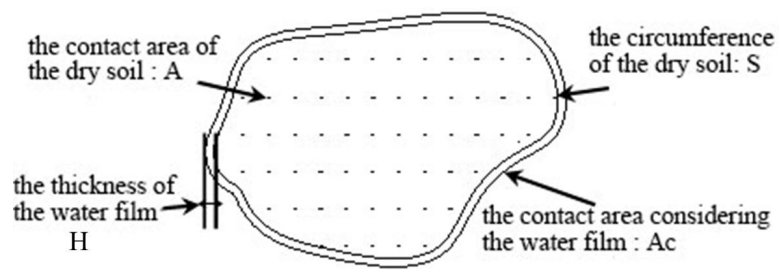

Fig. 5. The plan of particle contact area considering water film

Combining with equation (7), the relationship of the pore and the rate of the average pore area considering the water film thickness can be obtained. At the meantime, considering that the water film thickness varies with the properties of the soil, the water film thickness is set as $200 \AA, 250 \AA, 300 \AA, 350 \AA, 400 \AA$, and $450 \AA$ respectively. 
Statistical analysis is made to build the relationship of the porosity and the rate of average pore area, as shown in fig.6., fig.7. and fig.8..
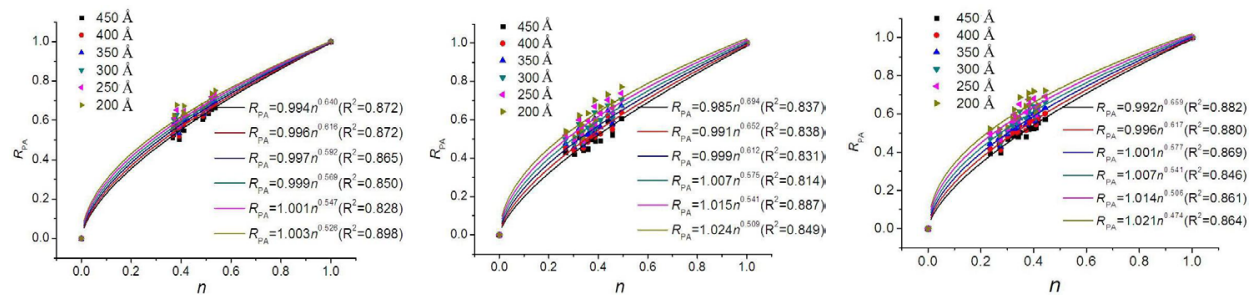

Fig. 6. $\sim$ Fig. 8. The $n \sim R_{P A}$ curve of Hangzhou, Ningbo, Wenzhou soft clay in different combined water film thickness

We can get the conclusion from these figures that the rate of average pore area considering the water film thickness of the three are consistent in changing trends. With the porosity becoming larger, the rate of the average pore area increases. The results indicate that their relationship can be well fitted by a power function. The equation is as follows:

$$
R_{C A}=1-R_{P A}=1-n^{\frac{2}{3} \alpha}
$$

In the equation, $R_{C A}$ is the rate of average contact area, and $R_{P A}$ is the rate of the average pore area. $\alpha$ is an undetermined parameters, which is related to the composition and the district of the clay.

\subsection{The static earth pressure considering the contact area of the particles by estimating water and earth pressures separately}

Assuming the earth fill on the back of the retaining wall is porous soil, based on principle of effective stress, the effective stress is transferred through the contact points between particles of soil. The lateral pressure on the retaining wall only exists on the surface where the soil contacts with the wall. Ignoring the effect of pore water, the pores are not able to transfer effective stress. But if the pore is full with water and the retaining wall is waterproof, the pore water pressure can be transferred to the retaining wall through the pore structure. According to the effective stress theory considering the contact area of the particles, based on the results of microstructure researches in the paper, the total stress acting on the retaining wall on unit length is as follows, if the soil on the back of the retaining wall is uniform and above the underground water level:

$$
E_{0}=\frac{1}{2} K_{0} \gamma H^{2}\left(1-n^{\frac{2}{3} \alpha}\right)
$$

In the equation above, $H$ is the height of the retaining wall, $n$ is the porosity of the soil and $\left(1-n^{\frac{2}{3} \alpha}\right)$ is the rate of particle contact area according to the microstructure study in this paper.

In practice, the effect of buoyancy arising from groundwater on the back of the retaining wall should be taken into consideration when calculating the static earth pressure. Utilizing the method of estimating water and earth pressures separately and submerged unit weight of soil $\left(\gamma^{\prime}\right)$ and considering the particle contact area and the pore area, we can get the soil pressure under water: 


$$
E_{0}=\frac{1}{2} K_{0} h_{2}^{2}\left(\gamma h_{1}+\gamma^{\prime} h_{2}\right)\left(1-n^{\frac{2}{3} \alpha}\right)+\frac{1}{2} K_{0} \gamma h_{1}^{2}\left(1-n^{\frac{2}{3} \alpha}\right)+\frac{1}{2} \gamma_{w} h_{2}^{2} n^{\frac{2}{3}}
$$

When the porosity of soil goes to zero, which means the soil is elastic solid material, equation (9) and (10) are the conventional formula of soil pressure calculation.

\section{Application example}

Calculate the static soil pressure and the resultant force on the retaining wall in Fig.9., where $h_{1}=2 \mathrm{~m}, h_{2}=4 \mathrm{~m}$. The basic physical parameters of the soil are listed in Table 1 .

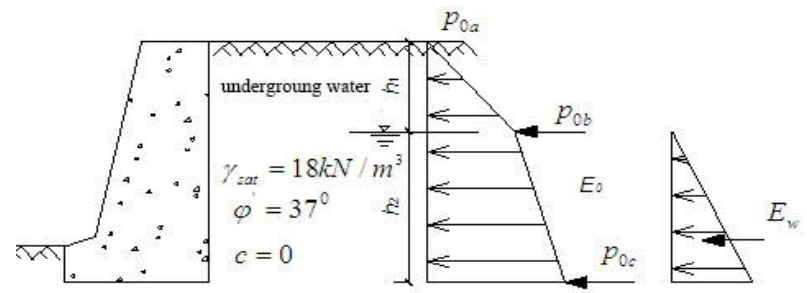

Fig. 9. Chart of earth pressure calculation

Table 1. Physical parameters of soft clay

\begin{tabular}{|c|c|c|c|c|c|}
\hline$g /\left(\mathbf{m} \cdot \mathbf{s}^{-2}\right)$ & $\gamma /\left(\mathbf{k N} \cdot \mathbf{m}^{-\mathbf{3}}\right)$ & $\gamma_{\text {sat }} /\left(\mathbf{k N} \cdot \mathbf{m}^{-\mathbf{3}}\right)$ & $\gamma^{\prime} /\left(\mathbf{k N} \cdot \mathbf{m}^{-\mathbf{3}}\right)$ & $c$ & $\varphi /\left(^{\circ}\right)$ \\
\hline 9.81 & 17.0 & 18.0 & 8.19 & 0 & 37 \\
\hline
\end{tabular}

The coefficient of earth pressure at-rest $K_{0}$ is:

$$
K_{0}=1-\sin \phi^{\prime}=1-\sin 37^{0}=0.4
$$

(1) The conventional method of estimating water and earth pressures together

At point a, $p_{0 a}=0$

At point b, $p_{0 b}=K_{0} \gamma h_{1}=0.4 \times 17 \times 2=13.6 \mathrm{kPa}$

At point c, $p_{0 c}=K_{0}\left(\gamma h_{1}+\gamma_{s a t} h_{2}\right)=0.4 \times(17 \times 2+18 \times 4)=42.4 \mathrm{kPa}$

The resultant force by this method is:

$$
\begin{aligned}
& E_{0}=\frac{1}{2}\left(p_{o a}+p_{o b}\right) h_{1}+\frac{1}{2}\left(p_{o b}+p_{o c}\right) h_{2} \\
& =\frac{1}{2} \times(0+13.6) \times 2+\frac{1}{2} \times(13.6+42.4) \times 4=125.6 \mathrm{kN} / \mathrm{m}
\end{aligned}
$$

(2) The conventional method of estimating water and earth pressures separately At point c, $p_{0 c}^{\prime}=K_{0}\left(\gamma h_{1}+\gamma^{\prime} h_{2}\right)=0.4 \times[17 \times 2+(18-9.81) \times 4]=26.7 \mathrm{kPa}$

The static water pressure on the wall:

$E_{w}=\frac{1}{2} \gamma_{w} h_{2}^{2}=\frac{1}{2} \times 9.81 \times 4^{2}=78.5 \mathrm{kN} / \mathrm{m}$ 
The resultant force is:

$$
\begin{aligned}
& E_{0}=\frac{1}{2}\left(p_{o a}+p_{o b}\right) h_{1}+\frac{1}{2}\left(p_{o b}+p_{o c}^{\prime}\right) h_{2}+E_{w} \\
& =\frac{1}{2}(0+13.6) \times 2+\frac{1}{2}(13.6+26.7) \times 4+78.5=172.7 \mathrm{kN} / \mathrm{m}
\end{aligned}
$$

(3) Estimating water and earth pressures separately considering the contact area

As is shown above, the results of the former two methods are not associated with the porosity and the parameter of the contact area. So assuming the soil is the clay in Hangzhou the paper do tests on, we set $n=0.4, \alpha=0.8$.

$$
\begin{aligned}
& E_{0}=\frac{1}{2}\left(p_{o a}+p_{o b}\right) h_{1}\left(1-n^{\frac{2}{3} \alpha}\right)+\frac{1}{2}\left(p_{o b}+p_{o c}^{\prime}\right) h_{2}\left(1-n^{\frac{2}{3} \alpha}\right)+E_{w} n^{\frac{2}{3} \alpha} \\
& =\frac{1}{2}(0+13.6) \times 2 \times\left(1-0.4^{\frac{2}{3} \times 0.8}\right)+\frac{1}{2}(13.6+26.7) \times 4 \times\left(1-0.4^{\frac{2^{2} \times 0.8}{3}}\right)+78.5 \times 0.4^{\frac{2^{2} \times 0.8}{3}} \\
& =84.57 \mathrm{kN} / \mathrm{m}
\end{aligned}
$$

Table 2. Results of calculation

\begin{tabular}{|c|c|}
\hline \multirow{2}{*}{ Methods } & Resultant force \\
\cline { 2 - 2 } & $E_{0} /(\mathbf{k N} / \mathbf{m})$ \\
\hline Estimating water and earth pressures together & 125.60 \\
\hline Estimating water and earth pressures separately & 172.70 \\
\hline $\begin{array}{c}\text { Estimating water and earth pressures separately considering the } \\
\text { contact area }\end{array}$ & 84.57 \\
\hline
\end{tabular}

The results above indicate that: the earth pressure reaches the maximum value which is $172.7 \mathrm{kN} / \mathrm{m}$ utilizing the conventional method of estimating water and earth pressures separately, followed by the value utilizing the conventional method of estimating water and earth pressures together, which is $125.6 \mathrm{kN} / \mathrm{m}$, and the result of estimating water and earth pressures separately considering the contact area comes minimum, which is $84.57 \mathrm{kN} / \mathrm{m}$.

\section{Conclusions}

Comparing to the conventional method of forefathers' research, the method of estimating water and earth pressures separately considering the effect of the contact area of the particles on the lateral stress transfer, put forward in this paper, is consistent with the calculation of point stress but differs mainly in the stress calculation on contact surface. In the research, the influence of the material contact area on earth pressure transfer is studied, with more rigorous principles of stress transfer and more comprehensive theoretical formulas, which satisfies the stress transfer characteristics of granular materials more scientific. The formula has its preconditions and enforcing validity. Because water pressures act on both sides of the support in hydrostatic condition, they need not to be taken into account when calculating the anti-sliding and anti-overturning stability. Meanwhile, as for the conditions the excess pore water pressure or seepage water pressure can't be ignored, the influence that positive negative characteristic of the excess pore water pressure as well as that of the flow direction have on seepage pressure should be taken into consideration. For example, during the excavation of foundation pit, unloading effects lead to movement 
of retaining structures and deep-level strata heave, and in this case, the pore water pressure is negative.

Instead of using the strength reduction method to calculate the pore water pressure, the method put forward in this paper considers the real contact area of the pore from the structural features of clay itself, which corresponds closely to the actual conditions in a sense. But the soil is much more complicated actually, and the distribution of the pore scale may not always be uniform. On account that the influence coefficient of the contact area $(\alpha)$ has influencing factors within itself, how to calculate the lateral earth pressure more accurately is remained to be further researched and verified in numerous engineering practices. The rationalization mind of earth pressure on the support structure provides important guarantee for the engineering construction. Moreover, in addition to the safety and economy, more aspects such as multi engineering conditions need to be taken into account comprehensively during the design. Namely it saves investment, satisfies the demands of the engineering structure and really comes to achieve the double winning goal of social and economic efficiency.

This work is supported by the National Natural Science Foundation of China (Grant No. 41672264).

\section{References}

1. T. Li, B. Liu, Y. Li, et al, The void ratio of saturated red clay calculated from a micro-structure model, J. Journal of China University of Mining and Technology. 40(5), 720-725 (2011)

2. N.K. Tovey, D.H. Krinsley, Mapping of the orientation of fine-grained minerals in soils and sediments, J. Bulletin of Engineering Geology \& the Environment. 46(1), 93-101 (1992)

3. Y. X. Wu. Quantitative approach on micro-structure of engineering clay, J. Bulletin of the Chinese academy of Geological Sciences. 23, 143-151 (1991)

4. R. ENRIQUE, H.S. PAUL, Microstructure investigation in unsaturated soils: a review with special attention to contribution of mercury intrusion porosimetry and environmental scanning electron microscopy, J. Geotechnical and Geological Engineering. 26(6), 705-727 (2008)

5. J.C. BAKER, B. GRABOWSKA-OLSZEWSKA, P.J.R. UWINS, ESEM study of osmotic swelling of bentonite from Radzionkow, J. Applied Clay Science. 9(6), 465-469 (1995)

6. Y. J. Chen, Y.F Wen, Water and earth pressures on the supporting structure around a foundation pit, J. Chinese Journal of Geotechnical Engineering. 21(2), 139-143 (1999)

7. G. X. Li, Estimating the water and earth pressures on the supporting structure around a foundation pit separately and together, J. Chinese Journal of Geotechnical Engineering. 22(3), 348-352 (2000)

8. B. J. Wang, B. Shi, Y. Cai. et.al, 3D visualization and porosity computation of clay soil SEM image by GIS, J. Chinese Journal of Geotechnical Engineering. 29(1), 251-255 (2008)

9. X. Z. Xu, P. C. Li, C. L. Li, Principle of effective stress based on porous medium, J. Mechanics in Engineering. 23(4), 42-45 (2001)

10. C.C. Chen, W. Sun, W. L. Zhou, Quantitative image analysis on inner damage of cementitious material, J. Journal of the Chinese Ceramic Society. 4, 574-580 (2010) 